\title{
Elucidation of the Genomic Nucleotide Sequence of Bovine Coronavirus and Analysis of Cryptic Leader mRNA Fusion Sites
}

VLADIMIR N. CHOULJENKO, TIMOTHY P. FOSTER, XIAOQING LIN, JOHANNES STORZ, AND KONSTANTIN G. KOUSOULAS

Department of Veterinary Microbiology and Parasitology, School of Veterinary

Medicine,Louisiana State University, Baton Rouge, LA 70803

\section{INTRODUCTION}

Over the last few years numerous respiratory bovine coronaviruses (RBCV) were isolated from respiratory infections of cattle including two shipping fever epizootics (Storz et al., 1996; Storz et al., 2000). The RBCV strain 98TXSF-110-LU (LU) and the enteric strain 98TXSF-110-ENT (ENT) were isolated from lung and intestinal samples, respectively, from an animal experiencing fatal respiratory disease during a 1998 shipping fever epizootic. We obtained and compared the entire genomic sequences of the LU and ENT isolates to delineate nucleotide (nt) and amino acid (aa) differences between them. In addition, we analyzed the leader-mRNA junction sequences of the $\mathrm{S}$ and $12.7 \mathrm{kDa}$ mRNAs specified by different BCV strains.

\section{MATERIALS AND METHODS}

The LU and ENT BCV strains were plaque purified, gradient purified, total RNA was extracted from infected HRT-18G cells, and cDNA was produced as described previously (Chouljenko et al., 1998). Primers for sequencing of unknown BCV RNA polymerase were designed based on the 
sequence of MHV-A59 c12 mutant (accession number AF 029248). To characterize the leader-mRNA junction sequences of the $\mathrm{S}$ and $12.7 \mathrm{kDa}$ mRNAs, individual junctions were amplified by RT-PCR, cloned into plasmid pCR 2.1 (Invitrogen, Inc, Carlsbad, CA) and sequenced. Primer LBCV annealing to the leader sequence in combination with primers $3 \mathrm{~B} 21$, $3 \mathrm{~B} 11$, and 3B26 annealing to coding sequences of S, were used to produce PCR products containing the putative leader-body mRNA junctions (Chouljenko and Kousoulas, 2000).

\section{RESULTS AND DISCUSSION}

\subsection{Genomic sequences of ENT and LU strains}

Sequence comparison between the intestinal and lung isolates derived from the same animal revealed that these viruses contained numerous single nucleotide substitutions. In general, the structure of the BCV RNA polymerase gene was found to be similar to the other coronaviruses. ORF la overlapped by $31 \mathrm{nt}$ with ORF $1 \mathrm{~b}$ located in the -1 reading frame with respect to the upstream ORF 1a. A total of 32 nt changes, 5 of which caused aa changes were detected within the predicted ORF la cDNA sequence between the LU and ENT isolates. Fifteen nt changes were detected within the ORF $1 \mathrm{~b}$ and none caused aa changes. Fifty-three nucleotide changes were detected between the LU and ENT strains, 17 of which caused amino acid changes located within a $9.5 \mathrm{~kb}$ sequence spanning all the nonpolymerase ORFs. Thus, genomic sequencing revealed that two genetically different BCV strains were isolated from lung and intestinal samples of the same animal.

\subsection{Intergenic leader-mRNA junctions of $S$ mRNA specified by different BCV strains}

Thirty cloned DNA fragments containing leader-body junction sequences of the S mRNA were obtained after RT-PCR using primer pair $\mathrm{LBCV} / 3 \mathrm{~B} 21$. The majority of these mRNA junctions utilized the canonical consensus sequence located within the $5^{\prime}$ UTR of S. Specifically, 29 of 30 clones contained the consensus intergenic sequence TCTAAAC as part of the $\mathrm{S}$ leader-mRNA junction (type 1, Fig. 1). One clone contained the noncanonical leader-body sequence TA located downstream from the initiation codon of $\mathrm{S}$ at nucleotide positions 63-64 (type 2; Fig. 1). 


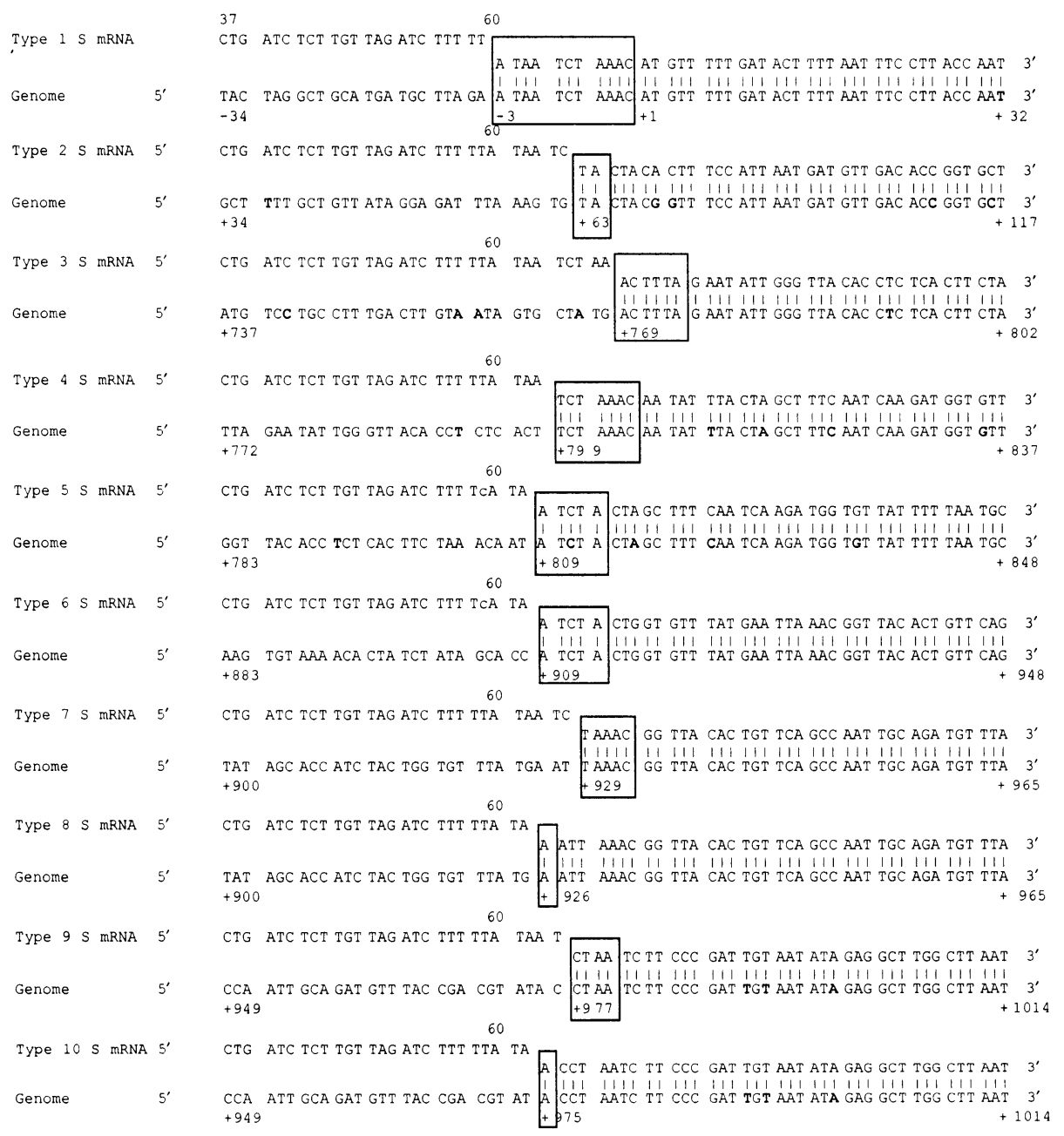

Figure 1. Cryptic leader-mRNA junction and flanking sequences of different mRNAs detected within the $5^{\prime}$ end of $S$. Leader and genome sequences are shown for the EBCV strain L9 except types 5 and 6 detected only for the RBCV OK strain (Chouljenko et al., 1998). Nucleotide sequences of subgenomic RNAs (sgRNAs) are shown in alignment with the genome sequence. Numbers above each sgRNA correspond to the leader sequence. Numbering on the genome sequence begins from the +1 (base $A$ of the start codon of S). The putative leader-body sequences are boxed. Numbers inside the boxes denote the first $\mathrm{nt}$ of the putative junction sequences counting from the start codon of $\mathrm{S}$. Nucleotide variations among different BCV strains noted previously (Chouljenko et al., 1998; Zhang et al., 1991) are shown as bold letters. The RBCV-specific change within the leader sequence is shown with a small letter. 


\subsection{Cryptic leader-mRNA junctions sequences within the 5 ' end of the S ORF}

The S ORF contains three heptameric nucleotide sequences that resemble the consensus intergenic (IG) sequence of S mRNA located within the 5' UTR (Zhang et al., 1991). The oligonucleotide primer 3B11 was used to specifically assess whether the intragenic IG homolog sequence located at nucleotide positions 799-805 of the $\mathrm{S}$ gene was utilized for the generation of mRNAs. A "shotgun" cloning of the heterogeneous LBCV/3B11 RT PCR products yielded a total of 33 DNA fragments encompassing $\mathrm{S}$ sequences ranging in size from 0.3 to $1.1 \mathrm{~kb}$. Five DNA fragments contained the predicted size of RT-PCR products, while the other 28 contained shorter junctions of different sizes. DNA sequence analysis of these junctions revealed 9 different types, all of which contained the leader fused to $S$ coding sequences (Fig. 1). The majority of the cloned DNA fragments represented type 4 junctions (14 clones) containing the targeted consensus sequence. The remaining junctions utilized nonconsensus sequences located both upstream and downstream from this consensus sequence analog. Similarly, different types of junction sequences were detected at the $3^{\prime}$ end of the S mRNA proximal to the other IG-like sequence, as well as within the 12.7 kDa ORF (Chouljenko and Kousoulas, 2000).

Overall, RT-PCR analysis using primer pairs that targeted two of the three intragenic consensus analog sequences located at the $5^{\prime}$ and $3^{\prime}$ ends of $\mathrm{S}$, respectively, confirmed that they were utilized for the production of mRNAs in infected cells. In addition, leader mRNA junctions containing nonconsensus sequences were also detected. Therefore, leader-mRNA junction formation is not strictly dependent on sequence homology between the $3^{\prime}$ end of the leader and the sequences of the mRNA fusion sites or on the location of these fusion sites within the mRNA. However, sequence complementarity seems to increase the efficiency by which homologous sites are used because canonical mRNAs are the predominant species detected by northern analysis (not shown).

Cryptic or alternative junction sequences were reported for a recombinant MHV mutant in which the green fluorescence protein (GFP) gene was inserted in the viral genome (Fischer et al., 1997). However, these junctions may have formed due to the GFP gene insertion within the MHV genome and in this regard, differ from our findings that cryptic junctions can be detected in infections with wild-type BCV strains.

Additional control experiments were performed to assure that the detected cryptic leader-mRNA fusion sequences were not produced by copy-choice template switching of the reverse transcriptase or Taq polymerase during the RT-PCR procedures. In these experiments, we selected the cell-adapted L9 and respiratory OK strains, because they contain a number of nucleotide differences within the genomic region bracketing the leader-mRNA junction sequences of the $12.7 \mathrm{kDa}$ ORF as 
well as a single nucleotide substitution between OK and L9 leader sequences. RNA was extracted from a control sample, which was prepared by mixing $1: 1$ virus stocks of strains $\mathrm{L} 9$ and $\mathrm{OK}$ containing approximate the same number of plaque forming units (PFU). Fourteen different DNA fragments were cloned and sequenced. Seven DNA fragments contained L9specific leader and part of the $12.7 \mathrm{kDa}$ body-mRNA sequences. Seven additional cloned DNA fragments contained OK-specific leader and bodymRNA, while two of these DNA fragments represented cryptic junction sequences. Overall, none of these cloned DNA fragments contained hybrid L9/OK sequences (not shown) proving that RT-PCR did not generate the observed cryptic junctions.

\subsection{Location of cryptic fusion sites on the predicted secondary structure of the S mRNA}

We generated the predicted global minimum energy secondary structure of the entire S mRNA using RNAdraw (Matzura and Wennborg, 1996). The predicted secondary structure of the BCV S mRNA revealed an unusually high level of internal homology causing extensive formation of double stranded RNA as well as multiple single stranded loops of RNA (Fig. 2). Interestingly, all cryptic leader-mRNA junctions as well as the junction within the $5^{\prime}$ UTR were predominantly located within single stranded portions of the S mRNA. Therefore, single stranded regions of mRNAs may be required for leader-mRNA junction formation.

\subsection{Single nucleotide substitutions proximal to junction sequences within the $S$ and $12.7 \mathrm{kDa} O R F$ specified by different virus isolates}

We found that different BCV strains contained a single nucleotide substitutions at the $5^{\prime}$ end of S, which coincided with the location of 6 cryptic in a total of 9 detected junctions (Fig. 1). In addition, a single nucleotide substitution was detected immediately proximal to the consensus heptameric sequence within the $3^{\prime}$ of the S ORF specified by RBCV strains associated with the 1998 Texas "shipping fever" epizootic, while the intestinal isolates remained unchanged (Chouljenko and Kousoulas, unpublished). Similarly, the intergenic region between the 4.9 and $12.7 \mathrm{kDa}$ ORFs, where the $12.7 \mathrm{kDa}$ ORF cryptic junctions were detected, is the most mutated area when RBCV strains are compared among them as well as with EBCV strains (Chouljenko et al., 1998). It is conceivable that the cryptic junction sequences also represent "hotspots" for recombination that may lead to sequence variation at these sites. 


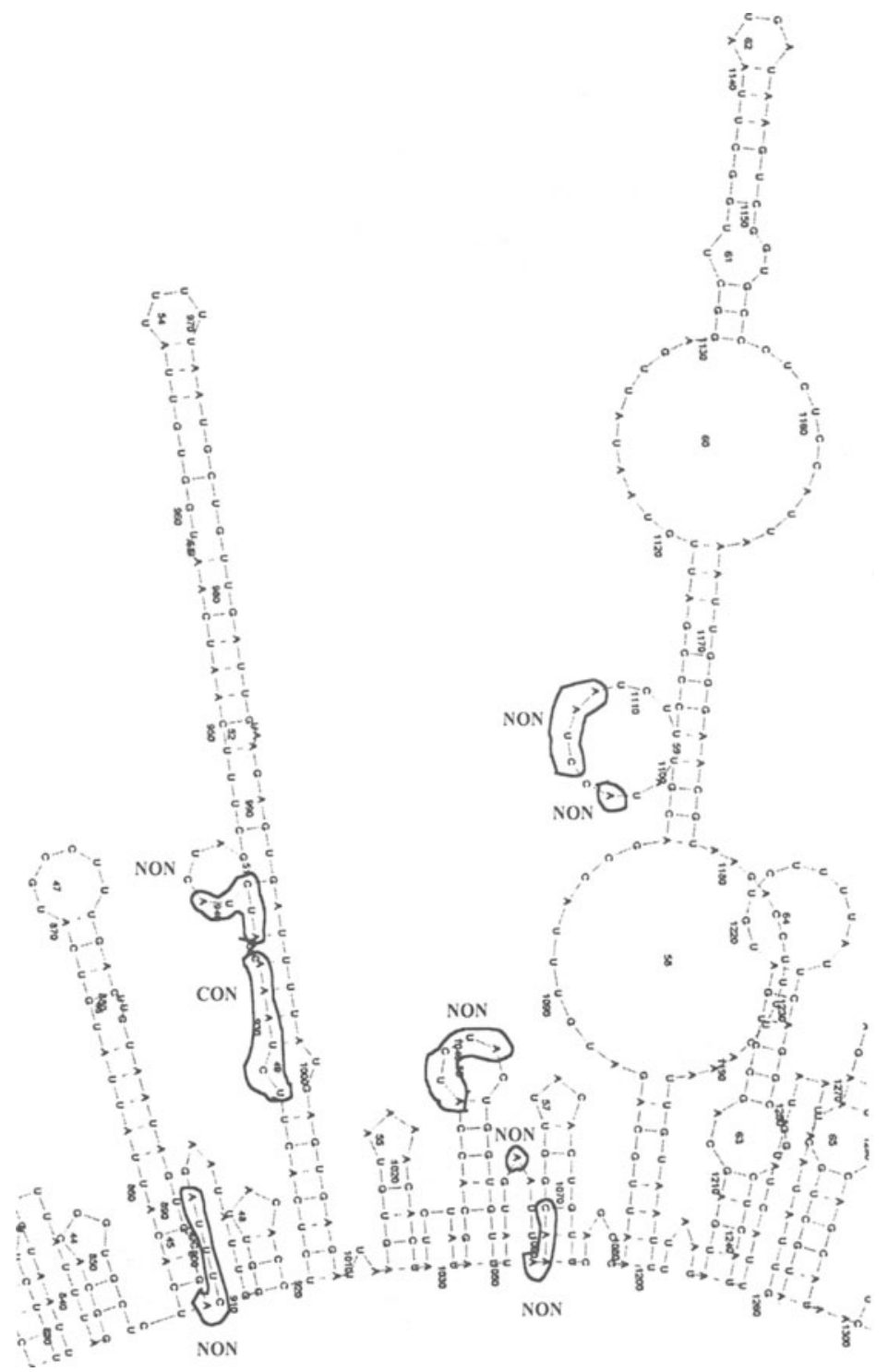

Figure 2. Predicted secondary structures of the S mRNA. A portion of the entire predicted secondary structure of the RBCV OK S mRNA is shown. Leader-mRNA body junctions utilizing the consensus and nonconsensus sequences are boxed and marked CON and NON, respectively. 


\section{REFERENCES}

Chouljenko, V. N., and K. G. Kousoulas. 2000. Cryptic leader-mRNA fusion sites within the bovine coronavirus $\mathrm{S}$ and $12.7 \mathrm{kDa}$ coding sequences (Submitted).

Chouljenko, V. N., K. G. Kousoulas, X. Lin, and J. Storz. 1998. Nucleotide and predicted amino acid sequences of all genes encoded by the 3 ' genomic portion $(9.5 \mathrm{~kb})$ of respiratory bovine coronaviruses and comparisons among respiratory and enteric coronaviruses. Virus Genes. 17:33-42.

Fischer, F., C. F. Stegen, C. A. Koetzner, and P. S. Masters. 1977. Analysis of a recombinant mouse hepatitis virus expressing a foreign gene reveals a novel aspect of coronavirus transcription. J. Virol. 71:5148-60.

Storz, J., C. W. Purdy, X. Lin, M. Burrell, R. E. Truax, R. E. Briggs, G. H. Frank, R. W. Loan. Isolation of respiratory bovine coronavirus, other cytocidal viruses, and Pasteurella spp from cattle involved in two natural outbreaks of shipping fever. J. Am. Vet. Med. Assoc. 216:1601-1606.

Storz, J., L. Stine, A. Liem, and G. A. Anderson. 1996. Coronavirus isolation from nasal swap samples in cattle with signs of respiratory tract disease after shipping. J. Am. Vet. Med. Assoc. 208:1452-5.

Zhang, X. M., K. G. Kousoulas, and J. Storz. 1991. Comparison of the Nucleotide and Deduced Amino Acid Sequences of the S-Genes Specified by Virulent and Avirulent Strains of Bovine Coronaviruses. Virology. 183:397-404.

Matzura, O., and A. Wennborg. 1996. RNAdraw:an integrated program for RNA secondary structure calculation and analysis under 32-bit Microsoft Windows. Comp. Applic. Bioscie. (CABIOS). 12:247-249. 